\title{
El cateterismo bilateral y simultáneo de los senos petrosos inferiores en el diagnóstico diferencial del síndrome de Cushing
}

\author{
Susana Belli ${ }^{1}$, Adriana 0 neto ${ }^{1 a}$, Esteban Mendaro². \\ Bilateral inferior petrosal sinus \\ sampling in the differential diagnosis of \\ ACTH-dependent Cushing's syndrome
}

Background: The features of pituitary ACTH-dependent Cushing syndrome are often indistinguishable from those of occult ectopic ACTH-dependent Cushing syndrome (CS). Aim: To assess the diagnostic accuracy of bilateral inferior petrosal sinus sampling (BIPSS) in the differential diagnosis of ACTH-dependent Cushing's syndrome as compared with ACTH levels and the overnight high dose dexamethasone suppression test (HDDST). Material and methods: Retrospective review of medical records of 23 patients (aged 19 to 63 years, 16 women) with surgically proven CS, 20 pituitary microadenomas (CD) and 3 with occult ectopic ACTH secretion (EAS). Results: No tumor was identifiable by imaging techniques. Mean plasma ACTH values were higher in patients with EAS than in CD $(103 \pm 110.2$ and $73.1 \pm 41.98 \mathrm{pg} / \mathrm{mL}$ respectively, $\mathrm{p}=\mathrm{NS})$. Three patients with EAS and 3 patients with CD did not suppress cortisol with the HDDST. The sensitivity of the test was $86 \%$ and the specificity 100\%. To improve the diagnostic outcome of BIPSS, an stimulation with Desmopressin (9 $\mu \mathrm{g}$ i.v.) was performed in 9 patients. The threshold for a pituitary source, was defined as an inferior petrosal sinus to peripheral ACTH basal and post Desmopression ratio $\geq 2$. BIPSS was successfully carried out in 22 patients and no complications occurred. In 6 patients BIPSS failed to meet the threshold criteria. In 3 patients, bronchial carcinoid tumors which proved to synthesize ACTH, were removed. The diagnostic sensitivity of BIPSS greatly improved from $86 \%$ to $100 \%$ after Desmopressin stimulation. BIPSS accurately predicted the lateralization of the microadenoma in 8 of 12 patients (66\%). Conclusions: The combination of Desmopressin stimulation with BIPSS was useful for the differential diagnosis of ACTH-dependent Cushing's Syndrome. However, the preoperative location of pituitary microadenomas was poorly predicted by BIPSS (Rev Méd Chile 2007; 135: 1095-102).

(Key words: Adrenocorticotropic hormone; Cushing syndrome; Deamino arginine vasopressin (Desmopressin); Petrosal sinus sampling)

Recibido el 30 de octubre, 2006. Aceptado el 20 de marzo, 2007.

${ }^{1}$ División Endocrinología del Hospital General de Agudos Dr. Carlos Durand. ${ }^{2}$ Hemodinamia y Angiografía Digital de Abraxas Médica, Clínica «a Sagrada Familia», Ciudad Autónoma de Buenos Aires, Argentina.

aBioquímica

Correspondencia a: Dra. Susana Belli. Av. Díaz Vélez 5044. (1405), Ciudad Autónoma de Buenos Aires, Argentina. Fax: (54)-011-6323-2940. E mail: susanabelli@speedy.com.ar 
$I^{d}$ dentificar la causa de un síndrome de Cushing dependiente de hormona adrenocórtico trófica (ACTH), es un paso esencial para elegir la terapéutica apropiada. La hipersecreción de ACTH es, en la mayoría de los pacientes, secundaria a un tumor hipofisario y aproximadamente en 10\% de los casos se origina en una fuente ectópica. Múltiples publicaciones ${ }^{1,2}$ han demostrado la limitada eficacia en el diagnóstico diferencial, tanto de las determinaciones basales de cortisol y de ACTH, como de las pruebas de supresión con dexametasona o de la estimulación con acetato de desmopresina, porque ambos tumores pueden responder de manera semejante. A su vez, tanto los microadenomas hipofisarios como los tumores ectópicos pueden, por su pequeño tamaño, no ser visibles con las técnicas radiológicas actuales ${ }^{3,4}$. Para superar estas limitaciones y confirmar 0 descartar el origen hipofisario de la enfermedad, en 1977 se desamolló el cateterismo bilateral y simultáneo de ambos senos petrosos inferiores, midiendo el ACTH que drenaba directamente de la hipófisis y estableciendo un gradiente de concentración con el ACTH de una vena periférica ${ }^{5}$. La eficiencia diagnóstica del procedimiento se incrementaba significativamente si se amplificaba el gradiente, estimulando la secreción de ACTH con la hormona liberadora $(\mathrm{CRH})^{6,7}$. Los pacientes con enfermedad de Cushing también aumentan la secreción de ACTH en respuesta al estímulo con el acetato de desmopresina, probablemente por la expresión anormal en el adenoma hipofisario de receptores V2 o V3. Esta característica puede ser utilizada durante el cateterismo, administrando acetato de desmopresina solo o asociado al $\mathrm{CRH}^{8,9}$.

Numerosas comunicaciones ${ }^{2,3,10}$ han sostenido que el cateterismo bilateral y simultáneo de los senos petrosos es el método más sensible para el diagnóstico diferencial entre el tumor hipofisario y el tumor ectópico secretante de ACTH, con 100\% de especificidad si el paciente está hipercortisolémico en el momento del procedimiento. Otra ventaja que se le ha asignado, es la posibilidad de localizar el adenoma dentro de la glándula, estableciendo un gradiente de la concentración de ACTH entre ambos senos $s^{8,10}$.

En la presente comunicación, presentamos nuestra experiencia con el cateterismo bilateral y simultáneo de los senos petrosos inferiores en pacientes con síndrome de Cushing ACTH depen- diente. Evaluamos la eficiencia diagnóstica del cateterismo antes y después de la administración de acetato de desmopresina. La comparamos con la sensibilidad de la determinación plasmática de ACTH y con la prueba de supresión nocturna con dosis altas de dexametasona. Analizamos si la existencia de un gradiente de ACTH entre ambos senos, fue capaz de localizar el tumor dentro de la glándula hipófisis antes de la cirugía.

\section{MATERIAL Y MÉTODO}

Desde 1988, realizamos el cateterismo bilateral y simultáneo de los senos petrosos inferiores en 23 pacientes con síndrome de Cushing ACTH dependiente que consultaron en forma consecutiva, en la División de Endocrinología del Hospital Durand, Ciudad Autónoma de Buenos Aires, Argentina. Dieciséis eran mujeres y 7 eran hombres, con una edad media de 33,5 $\pm 11,9$ años (19-63). Todos los pacientes sometidos al cateterismo de los senos petrosos tenían evidencia clínica de la enfermedad. El diagnóstico se confirmó por la presencia de niveles inapropiados y persistentemente elevados del cortisol plasmático y del cortisol libre urinario. Todos tenían ausencia del ritmo circadiano del cortisol y falta de inhibición del cortisol plasmático luego de la administración de $1 \mathrm{mg}$ de dexametasona nocturna. Se consideró que el síndrome de Cushing era ACTH dependiente, porque el ACTH plasmático era detectable a pesar del hipercortisolismo. A todos los pacientes se les realizó una tomografía computarizada/ resonancia magnética de silla turca, una tomografía computarizada de tórax y de abdomen con contraste y en ninguno se demostró la presencia de un tumor. En nuestra división, el cateterismo de los senos petrosos se indica sólo en aquellos pacientes con hipercortisolemia ACTH dependiente y sin evidencia radiológica de tumor, los que representan $60 \%$ de nuestra población.

El Comité de Docencia e Investigación del Hospital Durand aprobó la realización de este protocolo y todos los pacientes dieron su consentimiento informado para este procedimiento invasivo.

A partir de la comercialización en Argentina del acetato de desmopresina (DDAVP, análogo sintético de la arginina, Desmopresin ${ }^{\circledR}$ Ferring) en 9 cateterismos obtuvimos muestras de ACTH basales y 
posestímulo. Para determinar el origen hipofisario de la enfermedad, se estableció un gradiente de concentración de ACTH entre cada seno (SP) (derecho e izquierdo) y la periferia (P) (SP/P), en condiciones basales y con la concentración máxima de ACTH alcanzada luego de la administración de acetato de desmopresina. Utilizamos un valor de corte $\geq 2$, que fuera publicado previamente ${ }^{8,11}$, en el gradiente de ACTH seno/periferia, para el basal y posestímulo.

Para la localización intrahipofisaria del adenoma (lateralización: derecha o izquierda) utilizamos como valor de corte, un gradiente entre los senos $\geq 1,4$, que fuera publicado por otros autores ${ }^{12}$. Todos los pacientes que fueron clasificados como sospechosos de presentar una enfermedad hipofisaria, fueron operados por vía transeptoesfenoidal. Se consideró probada la enfermedad, si se extirpaba un tumor con alto contenido de ACTH por inmunohistoquímica o si el cortisol plasmático posquirúrgico era $<50 \mathrm{ng} / \mathrm{ml}$.

Evaluación bioquímica. A las 08:00 h, se obtuvo una muestra de sangre de una vena antecubital, para la determinación del cortisol sérico y del ACTH plasmático basales. A las 23:00 h, se les administraron $8 \mathrm{mg}$ de dexametasona por vía oral y se les realizó otra extracción a las 08:00 h del día siguiente para determinar la concentración de cortisol. Se consideró compatible con un origen hipofisario de la enfermedad, cuando la concentración de cortisol posdexametasona era menor 0 igual a $50 \%$ del valor basal ${ }^{13}$.

Técnica del cateterismo. Para evitar errores metodológicos derivados de un episodio de baja secreción de cortisol, les realizamos una determinación de cortisol previa al procedimiento. Bajo anestesia local se punzaron simultáneamente ambas venas femorales. Se utilizó un catéter Terumo ${ }^{\circledR}$ vertebral de 5 French con guía metálica de 0,035 pulgadas por 1,45 m, con el cual se realizó el cateterismo selectivo de la vena yugular interna y luego del seno petroso inferior en forma bilateral. Antes de obtener las muestras de sangre, se confirmó que los catéteres estaban correctamente ubicados en los senos petrosos inferiores con la inyección manual de contraste yodado. Se obtuvieron imágenes de frente y perfil del cráneo con substracción digital. Se consideró, que la ubicación de los catéteres era satisfactoria, si luego de la cateterización de ambos senos petrosos inferiores, se opacificaban correctamente el seno cavernoso o coronario homolateral. Con los catéteres correctamente ubicados en ambos senos petrosos, se obtuvieron muestras de sangre en forma simultánea de ambos senos petrosos y de la vena antecubital, antes (basal) y a los 2, 5 y 10 min posteriores a la administración endovenosa de $9 \mu \mathrm{g}$ de acetato de desmopresina. Una vez obtenidas las muestras, se realizó la compresión manual de las punciones femorales.

Recolección de las muestras. Durante el cateterismo, se extrajeron $2,5 \mathrm{ml}$ de sangre entera que se colocaron en un tubo con EDTA como anticoagulante para la determinación de ACTH. En los cateterismos sin estímulo, se procesaron 3 muestras basales (ambos senos y periferia) y 12 muestras en los cateterismos en los que utilizó acetato de desmopresina (basal, 2, 5 y $10 \mathrm{~min}$ de ambos senos y de la periferia). Las muestras se mantuvieron refrigeradas y se centrifugaron dentro de los 30 min de efectuada la extracción. El plasma se separó en viales de plástico y se lo congeló hasta su procesamiento.

Metodología. La concentración plasmática del cortisol fue determinada por radioinmunoensayo (RIA DSL-Texas). La sensibilidad del método fue de 5 $\mathrm{ng} / \mathrm{ml}$, con un coeficiente de variación inter e intraensayo de $8 \%$ y $9 \%$, respectivamente. El ACTH se dosó por el método radioinmunométrico IRMA (ELSA ACTH-CIS-Francia) ${ }^{14}$ hasta el año 2000 y a partir de entonces, por quimioluminiscencia (ICMA DPC-IMMULTE). El límite de detección funcional del IRMA es de $8 \mathrm{pg} / \mathrm{ml}$ y el del ICMA de $6 \mathrm{pg} / \mathrm{ml}$. Los coeficientes de variación inter e intraensayo fueron de $6 \%$ y $4 \%$ para el IRMA y de $7 \%$ y $5 \%$ para el ICMA, respectivamente.

Estadística. Los resultados se expresan en medias 土 desvío estándar. Utilizamos la prueba t para muestras no apareadas. Se consideró significativo un valor $\mathrm{p} \leq 0,05$. Se calcularon la sensibilidad $\mathrm{y}$ especificidad para cada prueba definiendo los casos como verdaderos negativos o positivos de acuerdo al resultado de la anatomía patológica. 


\section{RESULTADOS}

El procedimiento fue bien tolerado por todos los pacientes. La duración promedio de los cateterismos fue de $70 \mathrm{~min}$, se realizó en forma ambulatoria, con un promedio de estadía hospitalaria de 4 h. No observamos ninguna complicación, los pacientes sólo refirieron un disconfort transitorio en el oído durante la cateterización. De los 23 cateterismos realizados, 22 fueron técnicamente exitosos. La paciente restante presentaba una agenesia de senos petrosos, fue operada por vía transeptoesfenoidal y se le extirpó un adenoma hipofisario, tras lo cual remitió la enfermedad, por lo que se incluyen sus datos de laboratorio.
Gradiente de ACTH senos petrosos-periferia. En la Tabla 1, se detallan los valores individuales de ACTH basal y posestímulo con acetato de desmopresina y se lo grafica en la Figura 1. En 16/22 pacientes se observó un gradiente senos petrososperiferia $\geq 2$. De los 6 pacientes que no tuvieron gradiente, 5 tuvieron determinaciones de ACTH exclusivamente basales. A 3 de ellos que fueron operados por vía transeptoesfenoidal, se les resecó un adenoma hipofisario y la enfermedad remitió. En los otros 3 pacientes, se identificó posteriormente un tumor ectópico secretante de ACTH (carcinoide de pulmón) que también fue extirpado. En los pacientes 17 y 19 con tumor hipofisario, el estímulo con acetato de desmopre-

Tabla 1. C ateterismo de ambos senos petrosos inferiores.

\begin{tabular}{|c|c|c|c|c|c|c|}
\hline Paciente & $\begin{array}{l}\text { Seno Petroso } \\
\text { Izquierdo }\end{array}$ & $\begin{array}{l}\text { Seno Petroso } \\
\text { Derecho }\end{array}$ & Periferia & $\begin{array}{l}\text { Gradiente } \\
\text { SP/P }\end{array}$ & $\begin{array}{c}\text { Localización } \\
\text { quirúrgica } \\
\text { del tumor }\end{array}$ & $\begin{array}{c}\text { Gradiente } \\
\text { SPI/SPD }\end{array}$ \\
\hline 1 & 23-33-23-21 & 23-25-29-12 & $16-10-16-21$ & 1,6 & & \\
\hline 2 & 90 & 150 & 120 & 1,25 & & \\
\hline 3 & 230 & 260 & 220 & 1,18 & & \\
\hline 4 & 200 & 140 & 130 & 1,5 & & \\
\hline 5 & 38 & 50 & 38 & 1,3 & & \\
\hline 6 & 140 & 140 & 130 & 1,1 & & \\
\hline 7 & 9 & 97 & 10 & 9,7 & Derecho & 10,7 \\
\hline 8 & 200 & 1600 & 147 & 10,8 & Derecho & 8 \\
\hline 9 & 170 & 100 & 75 & 2,2 & & \\
\hline 10 & 150 & 1300 & 120 & 10,8 & Izquierdo & 8,6 \\
\hline 11 & 300 & 1700 & 170 & 10 & Derecho & 5,6 \\
\hline 12 & 150 & 800 & 150 & 5,3 & Central & 5,3 \\
\hline 13 & 130 & 48 & 40 & 3,5 & Central & 2,7 \\
\hline 14 & 72 & 860 & 66 & 13 & Derecho & 12 \\
\hline 15 & $120-110-200-155$ & $350-800-700-600$ & $12-52-80-12$ & 10 & & \\
\hline 16 & $50-45-85-60$ & $340-90-700-500$ & $50-40-52-62$ & 11,2 & Derecho & 8,2 \\
\hline 17 & 54-123-147-174 & $49-75-105-44$ & $56-53-55-54$ & 3,1 & Derecho & 1,2 \\
\hline 18 & $6-6-6-8$ & 82-101-230-77 & $17-16-16-16$ & 14 & Derecho & 28,7 \\
\hline 19 & 77-213-120-131 & 77-64-82-112 & 77-65-69-77 & 2,7 & & \\
\hline 20 & 287-88-98-78 & $900-541-452-198$ & $95-103-76-67$ & 8,7 & Derecho & 3,1 \\
\hline 21 & 1075-1306-1038-791 & 115-716-286-221-82 & $39-5-53-55-39$ & 23,7 & & \\
\hline 22 & $26-124-236-280$ & $326-3000-5600->6000$ & $30-23-142-170$ & 35,2 & Derecho & 21,4 \\
\hline
\end{tabular}

Concentración de ACTH en el seno petroso izquierdo, derecho y en una vena periférica. Basal, 2, 5 y 10 min posadministración endovenosa de $9 \mu \mathrm{g}$ de acetato de desmopresina. Gradiente de ACTH entre la máxima concentración en el seno petroso y en la periferia (SP/P). Gradiente de ACTH entre la máxima concentración en el seno izquierdo y en el derecho (SPI/SPD). Pacientes 1 a 3 con tumor ectópico secretante de ACTH. Pacientes 4 a 22 con tumor hipofisario. Pacientes 4 a 6 falsos negativos sólo con ACTH basal. 


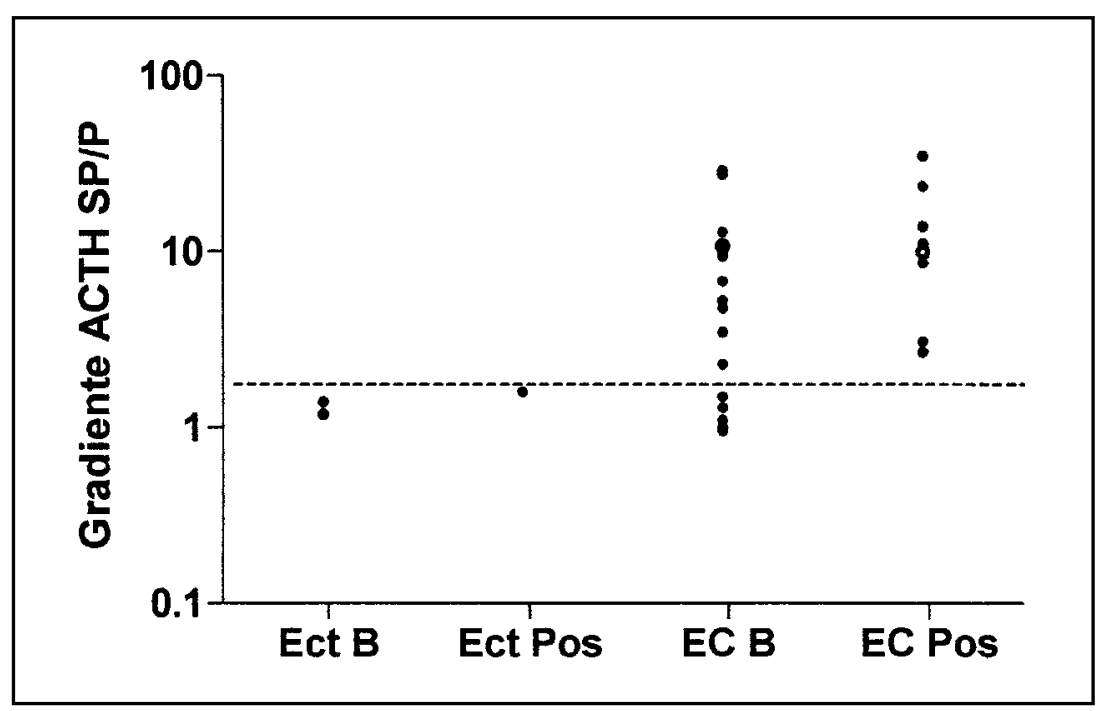

Figura 1. Máxima relación de la concentración ACTH seno petroso-periferia (SP/P) en los pacientes con síndrome de Cushing. La línea punteada indica el valor de corte $=2$ para el basal y posestímulo con acetato de desmopresina. Se denomina Ect: tumor ectópico secretante de ACTH. EC: enfermedad de Cushing. B: basal. Pos: postacetato de desmopresina.

sina desenmascaró un gradiente que no presentaban en las muestras basales $(0,96$ y 1 vs 3,1 y 2,7$)$. Para todo el grupo de pacientes con gradiente seno petroso/periferia mayor de 2 , los valores basales fueron entre 2,2 y 10,8, en tanto que luego del estímulo con desmopresina el gradiente se amplificó entre 2,7 y 35,2. La sensibilidad diagnóstica del cateterismo sólo con muestras basales fue de $86 \%$ y se incrementó a 100\% luego del estímulo con acetato de desmopresina, con una especificidad de $100 \%$.

Gradiente de ACTH entre ambos senos. Lateralización intrahipofisaria. Utilizando un valor de corte de ACTH basal o posestímulo $\geq 1,4$, hubo coincidencia entre la localización quirúrgica y el mayor gradiente, en 8 de los 12 pacientes (66\%).

Comparación entre la concentración de ACTH basal, el porcentaje de supresión del cortisol plasmático con $8 \mathrm{mg}$ de dexametasona nocturna y el gradiente de ACTH senos petrosos-periferia. Como puede verse en la Tabla 2, la concentración individual de ACTH en los pacientes portadores de un tumor ectópico (30-230 pg/ml) mostró una franca superposición con la de los pacientes con adenoma hipofisario (24-170 pg/ml). La concen- tración media de ACTH fue de 103 $\pm 110,2 \mathrm{pg} / \mathrm{ml}$ en los pacientes con tumor ectópico y de $73 \pm 41,98 \mathrm{pg} / \mathrm{ml}$ en los 20 pacientes con adenoma hipofisario. Si bien las medias eran diferentes, no alcanzaron significado estadístico. De los 20 pacientes con adenoma hipofisario, 17 presentaron una inhibición del cortisol basal $\geq 50 \%$. En los 3 pacientes que no inhibieron con dexametasona, el cateterismo confirmó el origen pituitario en 2, en la paciente restante las muestras de ACTH de los senos petrosos eran sólo basales. Ninguno de los pacientes con tumor ectópico secretante de ACTH inhibió con $8 \mathrm{mg}$ de dexametasona, lo que arroja para esta prueba una sensibilidad de $86 \%$ y una especificidad de 100\%. Tampoco mostraron gradiente en el cateterismo de los senos petrosos.

\section{DisCUSIÓN}

Uno de los mayores desafíos de la neuroendocrinología clínica es el diagnóstico diferencial entre una enfermedad hipofisaria y un tumor ectópico secretante de ACTH, utilizando las metodologías bioquímicas y radiológicas actuales. En coincidencia con lo referido por otros autores ${ }^{13,15}$, en nuestra población de pacientes, la concentración 
Tabla 2. Comparación entre la concentración plasmática del ACT H , el porcentaje de inhibición del cortisol plasmático con $8 \mathrm{mg}$ de dexametasona nocturno y el gradiente de ACTH seno petroso-periferia (SP/P)

\begin{tabular}{|cccc|}
\hline Paciente & $\begin{array}{c}\text { ACTH plasmática } \\
(\mathrm{pg} / \mathrm{ml})\end{array}$ & $\begin{array}{c}\text { Porcentaje de } \\
\text { inhibición }\end{array}$ & $\begin{array}{c}\text { Gradiente ACTH } \\
\text { SP/P }\end{array}$ \\
\hline 1 & 30 & -25 & 1,6 \\
2 & 50 & 21 & 1,25 \\
3 & 230 & 21 & 1,18 \\
4 & 100 & 66 & 1,5 \\
5 & 24 & 84 & 1,3 \\
6 & 36 & 48 & 1,1 \\
7 & 41 & 87 & 9,7 \\
8 & 65 & 98 & 10,8 \\
9 & 75 & 67 & 2,2 \\
10 & 65 & 60 & 10,8 \\
11 & 170 & 37 & 10 \\
12 & 150 & 87 & 5,3 \\
13 & 40 & 53 & 3,5 \\
14 & 65 & 92 & 13 \\
15 & 100 & 66 & 10 \\
16 & 38 & 86 & 11,2 \\
17 & 150 & 78 & 3,1 \\
18 & 73 & 93 & 14 \\
19 & 78 & 56 & 2,7 \\
20 & 36 & 73 & 8,7 \\
21 & 66 & 87 & 23,7 \\
22 & 30 & 44 & 35,2 \\
23 & 60 & 57 & Fracaso técnico \\
\hline
\end{tabular}

Pacientes 1 a 3 con tumor ectópico secretante de ACTH. Pacientes 4 a 22 con tumor hipofisario.

plasmática de ACTH mostró una importante superposición de los valores individuales, lo que no permitía un diagnóstico diferencial certero. La prueba de inhibición con dosis altas de dexametasona mostró 3 falsos negativos para el diagnóstico del tumor hipofisario, con una sensibilidad de $86 \%$ y una especificidad de $100 \%$. Estos hallazgos coinciden con la sensibilidad referida en la literatura, entre $59 \%$ y $92 \%$ y una especificidad entre $60 \%$ y $100 \% 1,15$. Nuestros 3 pacientes con tumor ectópico, como era de esperar, no inhibieron con dexametasona. Otros autores, sin embargo, han observado inhibición particularmente en tumores carcinoides de páncreas y de pulmón. Presumiblemente por la presencia de receptores a dexametasona en el tumor o por la secreción simultánea $\mathrm{ACTH} / \mathrm{CRH}$, que induciría la hiperplasia de las células corticotropas y sería finalmente la célula hipofisaria y no la célula tumoral, la respondiente a la dexametasona ${ }^{15}$.

El cateterismo de los senos petrosos para la medición directa del ACTH, aparece entonces, como el método más eficiente para el diagnóstico diferencial ${ }^{11}$. En nuestra población, 22 de 23 cateterismos fueron técnicamente exitosos y sólo 3 pacientes con adenomas hipofisarios quirúrgicamente demostrados no evidenciaron gradiente, representando 3 falsos negativos. En éstos, sólo obtuvimos muestras basales de ACTH, porque aún no se comencializaba el acetato de desmopresina en nuestro país. En los 9 pacientes que fueron estimulados con acetato de desmopresina no hubo falsos negativos, lo que aumentó significativamente la sensibilidad del procedimiento de $86 \%$ a $100 \%$. Nuestro estudio tiene limitaciones, el total de la población evaluada era pequeña y sólo tres pacien- 
tes tenían tumores ectópicos. Los datos de sensibilidad y especificidad fueron analizados teniendo en cuenta esta limitación. Es difícil superar esta traba, determinada por la baja prevalencia del síndrome de Cushing de origen ectópico, cuando se analizan los resultados de un único centro asistencial. Sólo estudios cooperativos multicéntricos, con un mayor número de casos, van a permitir precisar la sensibilidad del cateterismo de senos petrosos. A pesar de que la muestra es pequeña, nuestros hallazgos coinciden con lo referido por la mayoría de los autores para poblaciones más grandes, utilizando el estímulo con $\mathrm{CRH}$ solo o asociado al acetato de desmopresina ${ }^{8,11,19}$.

Otras causas de falsos negativos para el gradiente de ACTH incluyen: la localización ectópica del adenoma (ej: seno esfenoidal) que no drena al seno cavernoso, la presencia de un seno hipoplásico o plexiforme ipsilateral al microadenoma secretante de ACTH o el drenaje retrógrado a través de los senos portales del tallo hipofisario hasta el hipotálamo, diluyendo la sangre hipofisaria en la del seno petroso superior ${ }^{16,17}$. Por razones técnicas, como la ubicación incorrecta del catéter o por el manejo inadecuado de las muestras de sangre (hemólisis). También se han referido falsos negativos en tumores que secretan cíclicamente el ACTH y el procedimiento se realiza durante un episodio de baja secreción o durante la ingesta de drogas bloqueantes ${ }^{17}$. Estos errores se evitan confirmando que el paciente tiene el cortisol elevado en el momento del procedimiento (ej: cortisol libre en saliva la noche previa). Nosotros no observamos falsos positivos, pero éstos han sido ocasionalmente referidos en pacientes con tumores ectópicos secretantes de $\mathrm{CRH}$ sólo o concomitantemente con $\mathrm{ACTH}$. Por eso, en algunos centros miden la concentración periférica del $\mathrm{CRH}^{24}$.

\section{REFERENCIAS}

1. Aron DC, RafF H, Finding JW. Effectiveness versus efficacy: the limited value in clinical practice of high dose dexamethasone suppression testing in the differential diagnosis of adrenocorticotropin-dependent Cushing's syndrome. J Clin Endocrinol Metab 1997; 82: 1780-5.

2. Morris DG, Grossman AB. Dynamic tests in the diagnosis and differential diagnosis of Cushing's
En $66 \%$ de los pacientes operados, el cateterismo lateralizó correctamente el tumor dentro de la glándula en coincidencia con lo referido por otros autores que han comunicado una media de $75 \%$ y un rango entre $50 \%$ y $100 \% 13,8$. Para algunos investigadores ${ }^{8}$, la administración de $\mathrm{CRH}$ incrementó el valor predictivo positivo del cateterismo en la lateralización de $74 \%$ a $83 \%$, mientras que para el estudio multicéntrico italiano ${ }^{11}$ fue sorprendentemente menos eficiente que la resonancia magnética ( $65 \%$ vs $75 \%$ ).

En nuestras manos, en coincidencia con lo referido por la mayoría de los autores ${ }^{11,18}$, el cateterismo de los senos petrosos inferiores, fue un procedimiento seguro y bien tolerado a pesar de ser invasivo. Para los grupos con mayor número de casos realizados, el índice de éxitos y de complicaciones del cateterismo estaría directamente relacionado con la habilidad y el entrenamiento del operador, de allí que sólo debería realizarse en centros de probada experiencia ${ }^{7,8}$. Se han comunicado casos aislados de complicaciones de tipo neurológico ${ }^{19,20}$, tromboembolismo pulmonar $^{13}$ y trombosis venosa profunda en miembros inferiores ${ }^{21}$ con una prevalencia de alrededor de $0,2 \%$.

Creemos que los métodos complementarios de diagnóstico, aun los más sensibles para la identificación del tumor intrahipofisario, deben ser complementados con la elección de un cirujano experimentado en esta patología, que realice un examen minucioso de la glándula. El cateterismo bilateral y simultáneo de los senos petrosos con estímulo con acetato de desmopresina, fue un procedimiento seguro y sensible en el diagnóstico diferencial del síndrome de Cushing, pero fue poco eficiente para identificar la localización intrahipofisaria del adenoma.

syndrome. J Endocrinol Invest 2003; 26 (7 Suppl): 64-73.

3. Invitti C, Pecori Geraidi F, De Martin M, Cavagnin F, the Study Group of the ItaLan Society of EndocrinoLOgY on the Pathophysiology of the Hypothalamic Pituttary adrenal Axis. Diagnosis and management of Cushing Syndrome: results of an Italian multicenter study. J Clin Endocrinol Metab 1999; 84: 440-8.

4. Buchfelder M, Néstor R, Fahlbusch R, Huk WJ. The accuracy of CT and MR evaluation of the sella 
turcica for detection of adrenocorticotropic hormone- secreting adenomas in Cushing disease. Am J Neuroradiology 1993; 14: 1183-90.

5. Corrigan DF, SchaAf M, Whaley RA, Czerwinsky CL, EARL JM. Selective venous sampling to differentiate ectopic ACTH secretion from pituitary Cushing's syndrome. N Engl J Med 1977; 296: 861-2.

6. Landolt AM, Valavanis A, Girard J, Eberle AN. Corticotropin-releasing-factor test used with bilateral, simultaneous inferior petrosal sinus sampling in Cushing's syndrome. Adv Biosci 1988; 69: 361-2.

7. Findling JW, Aron DC,Tyrel JB, Shinsako JH, Fitzerald PA, Norman D, WiLson CB et al. Selective venous sampling for ACTH in Cushing's syndrome: differentiation between Cushing's disease and ectopic ACTH syndrome. Ann Intern Med 1991; 94: 647-52.

8. Kaltsas GA, Giannulis MG, Newel-Price JD, Dacie JE, Thakkar C, Afshar F et al. A critical analysis of the value of simultaneous inferior petrosal sinus sampling in Cushing's disease and the occult ectopic adrenocorticotropin syndrome. J Clin Endocrinol Metab 1999; 84: 487-92.

9. Newell-Price J, Perry L, Medback S, Monson J, SAVAge M, BesSER M et al. A combined test using desmopressin and corticotropin-releasing hormone in the differential diagnosis of Cushing's syndrome. J Clin Endocrinol Metab 1997; 82: 17681.

10. Oldfield EH, Doppman JL, Nieman LK Chrousos GP, Miler DL, Katz DA et al. Petrosal sinus sampling with and without corticotropin- releasing hormone for the differential diagnosis of Cushing's syndrome. N Engl J Med 1991; 325: 897-905.

11. Colao A, Faggiano A, Pivonello R Pecori Giraldi F, CaVagnini F, Lombardi G. Inferior petrosal sinus sampling in the differential diagnosis of Cushing's syndrome: results of an Italian multicenter study. Eur J Endocrinol 2001; 144: 499-507.

12. Oldfield EH, Chrousos GP, Schulte HM, Schaaf M, McKeever PE, Krudy AG et al. Preoperative lateralization of ACTH-secreting pituitary microadeno- mas by bilat eral and simultaneous inferior petrosal venous sinus sampling. N Engl J Med 1985; 312: 100-3.

13. Newell-Price J, Trainer P, Besser GM, Grossman AB. The diagnosis and differential diagnosis of Cushing' syndrome and pseudo-Cushing's states. Endocr Rev 1998; 19: 647-72.

14. Oneto A, Aranda C, Fisziejder L, Guitelman A, Schonholz C, Bew S. Utilidad clínica de la determinación de ACTH-IRMA. Resultados preliminares. Rev Argentina Endocrinol Metab 1992; 29: 8-14.

15. Arnaldi G, Angeli A, Atrinson B, Bertagna $X$, Cavagnini F, Chrousos GP et al. Diagnosis and complications of Cushing's Syndrome: a consensus statement. J Clin Endocrinol Metab 2003; 88: 5593-602.

16. Bergland RM, Page RB. Can the pituitary secrete directly to the brain? (affirmative anatomical evidence). Endocrinology 1978; 102: 1325-38.

17. Pecori Giraido F, Invitti $C$, Cavagnini F. Inferior petrosal sinus sampling ten years down the road. J Endocrinol Invest 2000; 23: 325-7.

18. Lefournier V, Martinie M, Vasdev A e Bessou $P$, Passagia JG, Labat-Moleur $F$ et al. Accuracy of bilateral inferior petrosal or cavernous sinuses sampling in predicting the lateralization of Cushing's disease pituitary microadenoma: influence of catheter position and anatomy of venous drainage. J Clin Endocrinol Metab 2003; 88: 196-203.

19. Yamamoto Y, Davis DH, Nippoldt TB, Young JR WF, Huston III J, PARISI JE. False positive inferior petrosal sinus sampling in the diagnosis of Cushing's disease. Report of two cases. J Neurosurg 1995; 83: 1087-91.

20. Miluer DL, Doppman JL, Peterman SB. Neurologic complications of petrosal sinus sampling. Radiology 1992; 185: 143-7.

21. Bonew FS, Huston III J, Carpenter PC, Erickson D, Young JR WF, MEYER FB. Adrenocorticotropic hormone dependent Cushing's syndrome: sensitivity and specificity of inferior petrosal sinus sampling. Am J Neuroradiol 2000; 21: 690-6. 